\title{
Global Rigidity: The Effect of Coning
}

\author{
R. Connelly • W.J. Whiteley
}

Received: 13 October 2008 / Revised: 6 August 2009 / Accepted: 7 August 2009 /

Published online: 28 August 2009

(C) Springer Science+Business Media, LLC 2009

\begin{abstract}
Recent results have confirmed that the global rigidity of bar-and-joint frameworks on a graph $G$ is a generic property in Euclidean spaces of all dimensions. Although it is not known if there is a deterministic algorithm that runs in polynomial time and space, to decide if a graph is generically globally rigid, there is an algorithm (Gortler et al. in Characterizing generic global rigidity, arXiv:0710.0907v1, 2007) running in polynomial time and space that will decide with no false positives and only has false negatives with low probability. When there is a framework that is infinitesimally rigid with a stress matrix of maximal rank, we describe it as a certificate which guarantees that the graph is generically globally rigid, although this framework, itself, may not be globally rigid. We present a set of examples which clarify a number of aspects of global rigidity.

There is a technique which transfers rigidity to one dimension higher: coning. Here we confirm that the cone on a graph is generically globally rigid in $\mathbb{R}^{d+1}$ if and only if the graph is generically globally rigid in $\mathbb{R}^{d}$. As a corollary, we see that a graph is generically globally rigid in the $d$-dimensional sphere $\mathbb{S}^{d}$ if and only if it is generically globally rigid in $\mathbb{R}^{d}$.
\end{abstract}

Keywords Infinitesimal rigidity $\cdot$ Global rigidity $\cdot$ Self-stress $\cdot$ Coning $\cdot$ Projective geometry $\cdot$ Spherical geometry

Research of the first author supported in part by NSF Grant No. DMS-0209595 (USA).

Work of the second author supported in part by a grant from NSERC (Canada).

R. Connelly ( $\varangle)$

Department of Mathematics, Cornell University, Ithaca, NY 14853, USA

e-mail: connelly@math.cornell.edu

W.J. Whiteley

Department of Mathematics and Statistics, York University, 4700 Keele Street, Toronto,

ON M3J 1P3, Canada

e-mail: whiteley@ mathstat.yorku.ca 


\section{Introduction}

Over the last 20 years, there has been a continuing development of methods and results in Global Rigidity [6, 7, 10, 12, 13]. Early work of Hendrickson provided clear necessary conditions for generic frameworks [12]: when the graph is not a simplex, it must be generically redundantly rigid (having a self-stress in each member) and $(d+1)$-vertex connected, in dimension $d$. The work of Connelly provided methods related to global minima of energy functions as well as some initial analysis of an example in [6].

Recent work has focused on specific techniques for global rigidity of generic frameworks. Connelly's work with stress matrices provided a sufficient condition for global rigidity of generic frameworks [7]. The paper also showed that the inductive technique of edge splitting preserves generic global rigidity in all dimensions. This in turn became a crucial technique in the work of Berg and Jordán [3] and Jackson and Jordán [13], which provided a proof that the necessary conditions of Hendrickson were also sufficient, in the plane. Later, they also provided an independent proof for this inductive technique, in the plane. As a final piece of the puzzle, Gortler, Healy, and Thurston [10] showed that, for generic configurations, Connelly's sufficient condition was also necessary, in all dimensions. As a corollary, if a framework is globally rigid for a generic configuration, then all generic configurations correspond to globally rigid frameworks. This property is combinatorial.

Knowing that generic global rigidity is combinatorial has increased the interest in global rigidity in general, and in finding additional combinatorial techniques for generating globally rigid frameworks $[4,8,14,15]$. Some of these papers provide some additional sufficient conditions in various dimensions that cover classes of frameworks which are studied in some applications. Among the current applications of global rigidity are location of sensors within a network [1,9] and general issues of uniqueness of constraints within CAD and computational geometry. At a recent workshop in Banff, there was a proposal that in the study of molecular rigidity and flexibility, global rigidity was the mathematical concept matching the stability of molecular structures, while infinitesimal rigidity matches fluctuation within a structure. These connections raise the overall interest in the study of global rigidity.

Given the difficulties of characterizing graphs which are generically infinitesimally rigid as bar-and-joint frameworks in 3-space [11, 19, 22], it is not a surprise that we have encountered difficulties with characterizing graphs which are generically globally rigid as bar-and-joint results in $d$-space. These difficulties are further emphasized by Connelly's proof [6] that the complete bipartite graph $K_{5,5}$ is not generically globally rigid in 3-space, although it satisfies the two necessary conditions of Hendrickson [7].

With these difficulties in giving overall characterizations of graphs which are generically globally rigid, there is added interest in new techniques which generate generically globally rigid graphs in higher dimensions. In Sect. 3 we show that particular properties of a specific framework $G(\mathbf{p})$ provide a certificate that the graph is generically globally rigid. Using this certificate, we use an algorithm, described in [10], which can demonstrate that a specific graph is generically globally rigid (Sect. 2). This is applied to a set of examples which demonstrate some key con- 
Fig. 1 Coning takes a framework in $d$-space to a framework in $(d+1)$-space, transferring redundant rigidity and taking $(d+1)$-connectivity to $(d+2)$-connectivity
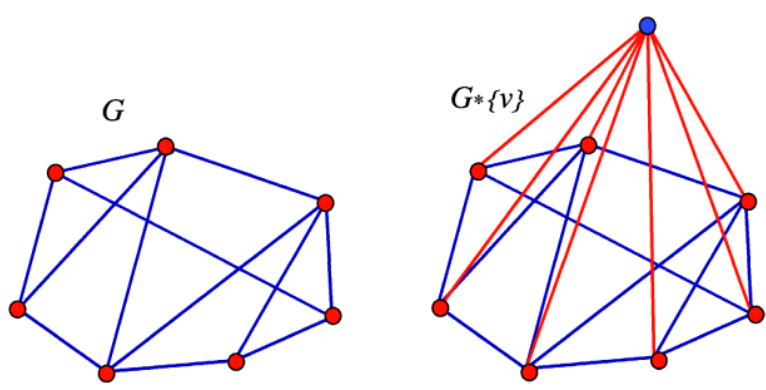

nections among global rigidity, the rank of the stress matrix, infinitesimal rigidity, and affine transformations in Sects. 3 and 8.

Coning (Fig. 1) is one process which is well studied for both geometric and generic rigidity [20]. This result is connected to the projective invariance of infinitesimal rigidity. In Sect. 5 we show directly that a graph is generically globally rigid in $\mathbb{R}^{d}$ if and only if the cone is generically globally rigid in $\mathbb{R}^{d+1}$. This proof is also connected to the invariance of the rank of the stress matrix (though not of global rigidity itself) under projective transformations (Sect. 4).

In Sect. 6 we convert this result on cones into a geometrically equivalent result for frameworks on the $d$-sphere $\mathbb{S}^{d}$. This shows that a graph $G$ is generically globally rigid in $\mathbb{R}^{d}$ if and only if $G$ is generically globally rigid in $\mathbb{S}^{d}$.

In Sect. 7 we outline another method that shows the equivalence of generic global rigidity in $\mathbb{S}^{d}, \mathbb{R}^{d}$, and $\mathbb{H}^{d}$, hyperbolic space, using a formula of Pogorelov in [16].

In Sect. 8 we present some additional examples, which further clarify the connections among the techniques and concepts in the paper. We also show that another set of properties of a specific framework $G(\mathbf{p})$ guarantees that there is an open neighborhood of $\mathbf{p}$ with all frameworks globally rigid and that the graph $G$ is generically globally rigid. In Sect. 9 we present some further conjectures and problems for future work.

Given the overall difficulty with characterizing generic global rigidity of bar-andjoint frameworks in dimensions greater than two, there is a general interest in the special class of frameworks which have good combinatorial characterizations for generic rigidity: the body-bar frameworks. In a separate paper with Tibor Jordán, we present a new result characterizing generic body-bar frameworks in all dimensions, by the simple necessary condition of generic redundant rigidity [8].

\section{Prior Results}

We recall the basic vocabulary and definitions from the literature [6, 7, 11, 22, 23].

A bar-and-joint framework in $\mathbb{R}^{d}$ is a graph $G$ and a configuration $\mathbf{p}$ which assigns a point $\mathbf{p}_{i}$ in $R^{d}$ to each vertex $i$ of the graph. For each edge $\{i, j\} \in E, \mathbf{p}_{i} \neq \mathbf{p}_{j}$.

Given a framework $G(\mathbf{p})$ in $\mathbb{R}^{d}$, an infinitesimal motion $\mathbf{p}^{\prime}$ is a solution to the system of linear equations $\left(\mathbf{p}_{i}-\mathbf{p}_{j}\right) \cdot\left(\mathbf{p}_{i}^{\prime}-\mathbf{p}_{j}^{\prime}\right)=0$. This system of equations is brought together as $R_{G}(\mathbf{p})\left(\mathbf{p}^{\prime}\right)^{T}=0$, with the $|E| \times d|V|$ rigidity matrix $R_{G}(\mathbf{p})$. An 
infinitesimal motion is trivial if it is the derivative of an analytic path of congruences, and nontrivial otherwise. A framework $G(\mathbf{p})$ is infinitesimally rigid if all infinitesimal motions are trivial, and infinitesimally flexible otherwise.

A framework $G(\mathbf{p})$ is globally rigid in $\mathbb{R}^{d}$ if all frameworks $G(\mathbf{q})$ in $\mathbb{R}^{d}$ which are $G(\mathbf{p})$-equivalent (have all bars the same length as $G(\mathbf{p})$ ) are congruent to $G(\mathbf{p})$.

A configuration is generic if the coordinates do not satisfy any nonzero polynomial equation with integer coefficients (or equivalently algebraic coefficients). It is important to realize that the generic configurations are not an open set of configurations in $\mathbb{R}^{d|V|}$, but they do form a dense set of full measure.

Hendrickson [12] proved two key necessary conditions for global rigidity of a framework at a generic configuration.

Theorem 1 (Hendrickson [12]) If a framework $G(\mathbf{p})$, other than a simplex, is globally rigid for a generic configuration $\mathbf{p}$ in $\mathbb{R}^{d}$, then:

- The graph $G$ is vertex $(d+1)$-connected.

- The framework $G(\mathbf{p})$ is redundantly rigid, in the sense that removing any one edge leaves a graph which is infinitesimally rigid.

A graph $G$ is generically globally rigid in $\mathbb{R}^{d}$ if $G(\mathbf{p})$ is globally rigid at all generic configurations $\mathbf{p}[6,7]$. It is now known that global rigidity is a generic property in this sense for graphs in each dimension [7, 10]. The critical technique used for proving global rigidity of frameworks uses stress matrices. This technique is at the core of the proof that global rigidity is a generic property, as well as some specific inductive techniques (below).

Clearly, the conditions of Theorem 1 are necessary for generic global rigidity. They are also sufficient on the line and in the plane [13]. However, by a result of Connelly [6], $K_{5,5}$ in 3-space is generically redundantly rigid and 5-connected, but is not generically globally rigid. See Example 3.1 .

This stress matrix approach was started in [5] and builds on the fact that any globally rigid framework, except for a simplex, is dependent with a self-stress $\omega$. Recall that a self-stress is an assignment of scalars $\omega_{i j}$ to the edges such that for each $i \in V$,

$$
\sum_{\{i j \in E\}} \omega_{i j}\left(\mathbf{p}_{i}-\mathbf{p}_{j}\right)=\mathbf{0} .
$$

This can also be seen as a linear dependence of the rows of the rigidity matrix [22].

Given a stress, there is an associated $|V| \times|V|$ symmetric matrix $\Omega$, the stress matrix such that for $i \neq j$, the $i, j$ entry of $\Omega$ is $-\omega_{i, j}$, and the diagonal entries for $i, i$ are $\sum_{j \neq i} \omega_{i, j}$. Note that all row and column sums are now zero, and Connelly has developed a number of properties of these stress matrices $[6,7]$.

The key connection for global rigidity is the following pair of results:

Theorem 2 (Connelly [7]) If $\mathbf{p}$ is a generic configuration in $\mathbb{R}^{d}$ such that there is an equilibrium stress where the rank of the associated stress matrix $\Omega$ is $|V|-d-1$, then $G(\mathbf{p})$ is globally rigid in $\mathbb{R}^{d}$. 
Theorem 3 (Gortler, Healy, and Thurston [10]) Suppose that $\mathbf{p}$ is a generic configuration in $\mathbb{R}^{d}$ such that $G(\mathbf{p})$ is globally rigid in $\mathbb{R}^{d}$. Then either $G(\mathbf{p})$ is a simplex or there is an equilibrium stress where the rank of the associated stress matrix $\Omega$ is $|V|-d-1$.

These results and their corollaries provide us with the basic tools for the analysis which follows.

\section{Computing Global Rigidity}

The results of Sect. 2 are stated for configurations $\mathbf{p}$ that are generic. Although the notion of being generic means that there is a (countably) infinite number of constraints to avoid, it turns out that there only is a finite number of polynomial conditions on the coordinates of $\mathbf{p}$ that must be avoided for $G(\mathbf{p})$ to be globally rigid. But for infinitesimal rigidity, a description of what these conditions are turns out to be difficult and particularly for global rigidity, a description of what these conditions are turns out to be almost impossible to determine in a useful way. Implicitly in [7] and more explicitly in [10], the following results can be used to determine generic rigidity and generic global rigidity.

Recall that a function $f: \mathbb{R}^{d} \rightarrow \mathbb{R}$ is lower semi-continuous at $\mathbf{p} \in \mathbb{R}^{d}$ if, for every $\epsilon>0$, there is a $\delta>0$ such that, for all $|\mathbf{q}-\mathbf{p}|<\delta, f(\mathbf{q}) \geq f(\mathbf{p})-\epsilon$. Loosely speaking, lower semi-continuity means that the function $f$ can jump up, but not down, near a given point $\mathbf{p}$. The following is an easy consequence of the basic properties of the rank of a matrix.

Lemma 4 The rank of a finite matrix is lower semi-continuous in its coordinates.

We can now use nongeneric configurations to determine rigidity and global rigidity at generic configurations.

Theorem 5 If $\mathbf{p}$ is a configuration in $\mathbb{R}^{d}$ such that $G(\mathbf{p})$ is infinitesimally rigid and if there is an equilibrium stress where the rank of the associated stress matrix $\Omega$ is $|V|-d-1$, then $G$ is generically globally rigid in $\mathbb{R}^{d}$.

Proof Infinitesimal rigidity of a framework is determined when the rank of the rigidity matrix $R(\mathbf{p})$ is maximal for configurations $\mathbf{p}$ in $\mathbb{R}^{d}$. By Lemma 4 , for any configuration $\mathbf{q}$ sufficiently close to $\mathbf{p}, G(\mathbf{q})$ will also be infinitesimally rigid in $\mathbb{R}^{d}$. In other words, the rank of $R(\mathbf{q})$ remains constant in a neighborhood $U$ of $\mathbf{p}$. So a basis for the stresses $\omega$ for $R(\mathbf{q})$ can be written so that the coordinates of each basis element of $\omega$ are rational functions of $\mathbf{q}$ defined on $U$. Thus there is a stress for $G(\mathbf{q})$ whose stress matrix $\Omega^{\prime}$ has its coefficients arbitrarily close to the stress matrix $\Omega$ for $G(\mathbf{p})$, which had rank $|V|-d-1$. By Proposition 1.2 of [7], since the dimension of the affine span of $\mathbf{q}$ is $d$, the rank of $\Omega^{\prime}$ is at most $|V|-d-1$. Again by Lemma 4, for $\mathbf{q}$, the rank of $\Omega^{\prime}$ is $|V|-d-1$ as well. We may choose $\mathbf{q}$ generic, and so by Theorem 2, $G(\mathbf{q})$ is globally rigid. By Corollary 1.14 in [10], $G(\mathbf{q})$ is globally rigid at all generic configurations $\mathbf{q}$. 
With this in mind we say that a configuration $\mathbf{p}$ with a stress $\omega$ for a graph $G$ is a certificate for generic global rigidity if the rank of the rigidity matrix $R(\mathbf{p})$ is $d|V|-d(d+1) / 2$ and the corresponding stress matrix $\Omega$ has rank $|V|-d-1$. So Theorem 5 says that if a graph has a certificate for generic global rigidity, it is generically globally rigid. Bear in mind, though, that if $\mathbf{p}$ and $\omega$ form a certificate for generic global rigidity for the graph $G, G(\mathbf{p})$ itself may not be globally rigid. See the examples of Sect. 8 .

The problem remains to find a combinatorial, or at least a discrete, calculation to determine generic rigidity and generic global rigidity. The following result and the resulting algorithm is one case of a result in [10], where it is shown not just for the field $\mathbb{Z}_{p}$ but also for the integers $\mathbb{Z}$.

Corollary 6 (Gortler-Healy-Thurston [10]) Let a real prime number $p$ be given. If $\mathbf{p}$ is a configuration in $\mathbb{Z}_{p}^{n}$ such that $G(\mathbf{p})$ is infinitesimally rigid (i.e., the rigidity matrix has rank $|V| d-\left(\begin{array}{c}d+1 \\ 2\end{array}\right)$ for $\left.|V| \geq d+2\right)$ and if there is an equilibrium stress where the stress matrix $\Omega(\bmod p)$ has rank $|V|-d-1$, then $G$ is generically globally rigid in $\mathbb{R}^{d}$.

Proof Let $\tilde{\mathbf{p}}$ be a configuration with integer entries, where each coordinate is congruent to the corresponding entry of $\mathbf{p}(\bmod p)$. The rank of the rigidity matrix $R(\tilde{\mathbf{p}})$ is also maximal, since a $[d n-d(d+1) / 2]$-dimensional sub-determinant is nonzero $(\bmod p)$ and thus it is nonzero in $\mathbb{R}$. Consider the rational stresses for the framework $G(\tilde{\mathbf{p}})$. They are solutions to a system of linear equations with integer coefficients, and the rank of this linear system is the same as the rank of the corresponding linear system in the field $\mathbb{Z}_{p}$, since the rank is again determined by a sub-determinant. If some appropriate subset of the stresses is fixed, then there is a unique solution for the remaining stresses $(\bmod p)$. By multiplying all the stresses by an appropriate integer, which is nonzero $(\bmod p)$, we get an integer solution for the stresses, and up to the nonzero $(\bmod p)$ scaling factor, this integer solution $\tilde{\Omega}$ yields the same stress matrix $(\bmod p)$ as $\Omega$, since both solutions are given by Cramer's Rule, for example. Thus the rank of $\tilde{\Omega}$ is $|V|-d-1$, the same as the rank of $\Omega$. Finally, $G(\tilde{\mathbf{p}})$ satisfies the conditions of Theorem 2 , and $\tilde{\mathbf{p}}$ and the integral stress $\tilde{\omega}$ are a certificate for $G$ being generically globally rigid in $\mathbb{R}^{d}$.

This leads to the following algorithm to determine generic global rigidity in $\mathbb{R}^{d}$ for an abstract graph $G$.

1. Choose a prime number $p$. (This should be such that $p^{d}$ is sufficiently larger than $|V|$.)

2. Choose a random integer configuration $\mathbf{p}(\bmod p)$ in $\mathbb{Z}_{p}^{n}$.

3. Solve the equilibrium equations $(\bmod p)$ for the linear system of stresses for $G(\mathbf{p})$ $(\bmod p)$. If the dimension of this system is not $[|E|-d|V|-d(d+1) / 2 \geq 1]-$ dimensional, where $|E|$ is the number of edges of $G$, either, go back to 1 . or declare that $G$ is probably not even redundantly rigid.

4. Choose a random stress matrix $\Omega(\bmod p)$ from the system of stresses for $G(\mathbf{p})$. If the rank of $\Omega=|V|-d-1$, stop and declare that $G$ is generically globally rigid 
in $\mathbb{R}^{d}$. If not, then either go back to 2 . or declare that $G$ is probably not generically globally rigid in $\mathbb{R}^{d}$.

The following are some examples, where this algorithm has been applied:

Example 3.1 The complete bipartite graph $K_{5,5}$ in $\mathbb{R}^{3}$ : The rank of $\Omega$ turns out to be 2 , which suggests that $K_{5,5}$ is not globally rigid in $\mathbb{R}^{3}$ even though it is redundantly rigid and vertex 4-connected. Indeed, an application of the results in [2] shows that this is the case for a generic configuration. So by Theorem $3, K_{5,5}$ is not globally rigid in $\mathbb{R}^{3}$. In [6], Theorem 3 was not available, and another method was used to show that $K_{5,5}$ is not generically globally rigid $\mathbb{R}^{3}$.

Example 3.2 The complete bipartite graph $K_{5,5}$ in $\mathbb{R}^{3}$ with an edge removed and replaced by an edge between two vertices in one of the partitions: The rank of $\Omega$ turns out to be $6=10-3-1=|V|-d-1$, and the graph is infinitesimally rigid. Thus this graph is generically globally rigid in $\mathbb{R}^{3}$.

Example $3.3 K_{5,5}$ in $\mathbb{R}^{3}$ with one edge split: This subdivides an edge, adding a new vertex and connecting it to two other vertices. $|V|=11$, and the rank of the stress matrix is $7=11-3-1$. Thus this graph is generically globally rigid in $\mathbb{R}^{3}$.

Example 3.4 The cone $K_{5,5} *\{v\}$ in $\mathbb{R}^{4}$. Here it seems that the rank of the stress matrix remains 2 , and thus it appears that $\{v\} * K_{5,5}$ in $\mathbb{R}^{4}$ is not generically globally rigid, although it is infinitesimally rigid. Indeed, it is not generically globally rigid, and this is described in Sect. 5.

\section{Projective Transformations}

One of the fundamental operations on the configuration of a framework, which preserves its infinitesimal rigidity, is a projective transformation. Here we show that a projective transformation also preserves the rank of a stress matrix that is associated to configuration $\mathbf{p}$ in $\mathbb{R}^{d}$. In the process, we will set up the tools that we will need in the next section to study the effects of coning.

For any configuration $\mathbf{p}$ in $\mathbb{R}^{d}$, define the $d$-by- $|V|$ configuration matrix $P=$ $\left[\mathbf{p}_{1}, \ldots, \mathbf{p}_{|V|}\right]$, where each $\mathbf{p}_{i}$ is regarded as a column vector. Similarly define the $(d+1)$-by- $|V|$ augmented configuration matrix $\hat{P}=\left[\hat{\mathbf{p}}_{1}, \ldots, \hat{\mathbf{p}}_{|V|}\right]$, where each $\hat{\mathbf{p}}_{i}$ is $\mathbf{p}_{i}$ with an additional bottom entry 1 . It is easy to show (see [7]) that the equilibrium condition (1) holds for the stress associated to a stress matrix $\Omega$ if and only if $\hat{P} \Omega=\mathbf{0}$.

With this terminology, we see that a configuration with corresponding augmented configuration matrix $\hat{Q}$ is projectively equivalent to the configuration corresponding to the augmented configuration matrix $\hat{P}$ if and only if there is a $(d+1)$-by- $(d+1)$ matrix $A$ such that $A \hat{P}=Q$, where the columns of $\hat{P}$ and $Q$ correspond to the configurations in homogeneous coordinates. The bottom row of $Q$ may not be all 1's, so $Q$ may not be an augmented configuration matrix. So let $\lambda_{1}, \ldots, \lambda_{|V|}$ be the bottom row of $Q$. We assume that none of these $\lambda_{i}$ are zero. (Any 0 entry corresponds to 
a vertex at infinity.) Let $D$ be the $|V|$-by- $|V|$ diagonal matrix with diagonal entries $\lambda_{1}, \ldots, \lambda_{|V|}$. Then $Q D^{-1}=\hat{Q}$ is an augmented configuration matrix corresponding to a configuration that is the projective image of the configuration corresponding to $\hat{P}$. Thus we get the following:

Proposition 7 Suppose that the configuration $\mathbf{q}$ is a projective image of the configuration $\mathbf{p}$ in $\mathbb{R}^{d}$ and that $\Omega$ is a stress matrix corresponding to a stress for $\mathbf{p}$. Then $D \Omega D$ is a stress matrix for the configuration $\mathbf{q}$, for an appropriate invertible diagonal matrix $D$.

Proof Following the discussion above, since $\hat{P} \Omega=\mathbf{0}$,

$$
\hat{Q} D \Omega D=A \hat{P} D^{-1} D \Omega D=A \hat{P} \Omega D=\mathbf{0} .
$$

From this result and from the classical results for infinitesimal rigidity, we have a key, but subtle, invariance. By this result, the rank of the stress matrix is invariant under projective transformation, as is the rank of the rigidity matrix. So if $G(\mathbf{p}), \omega$ is a certificate for generic global rigidity, then so is $G(\hat{\mathbf{p}}), \hat{\omega}$. However, the global rigidity of a specific configuration is not an invariant even under affine transformations. See Example 8.3 for further refinements of this invariance.

\section{Coning}

Coning is well recognized as a method for preserving infinitesimal rigidity and generic rigidity, between dimensions [20]. Recall that coning a graph $G$ adds a new vertex $v$, and adds edges from $v$ to all the original vertices in $G$, creating the cone graph $G *\{v\}$. Given a configuration $\mathbf{p} *$ for the cone graph in $\mathbb{R}^{d+1}$ such that the cone vertex $\mathbf{u}$ is distinct from the vertices of $G$ and such that the line through $\mathbf{u}$ and $\mathbf{p}_{i}$ intersects a hyperplane $H \cong \mathbb{R}^{d}$, then $\mathbf{p}_{H}$ is the projection of $\mathbf{p} *$ from the cone vertex $\mathbf{u}$ into $H$ (Fig. 2). We call $\mathbf{p} *, \mathbf{p}_{H}$ a projection pair of configurations.

The following basic result confirmed a piece of the engineering folklore in rigidity theory which was passed into our mathematical community by the geometer and engineer Janos Baracs.

Fig. 2 Moving vertices radially in and out on the cone does not change infinitesimal rigidity or global rigidity
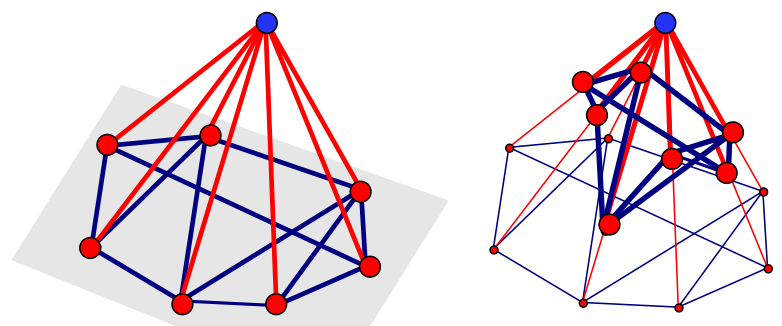
Theorem 8 (Infinitesimal Rigidity Coning [20]) Given a graph $G$, a cone graph $G *$ $\{v\}$, and a projection pair of configurations $\mathbf{p} *, \mathbf{p}_{H}$, then $G *\{v\}(\mathbf{p} *)$ is infinitesimally rigid (resp. independent) in $\mathbb{R}^{d+1}$ if and only if $G\left(\mathbf{p}_{H}\right)$ is infinitesimally rigid (resp. independent) in $\mathbb{R}^{d}$.

We now prove the following, which is the main result about coning.

Theorem 9 Given a graph $G$, a cone graph $G *\{v\}$, and a projection pair of configurations, then the maximum rank of a stress matrix of $G\left(\mathbf{p}_{H}\right)$ in $H$ is equal to $|V|-d-1$ if and only if the maximum rank of the stress matrix of $G *\{v\}(\mathbf{p} *)$ in $\mathbb{R}^{d+1}$ is also $|V|-d-1$, where $|V|$ is the number of vertices of $G$, and $d$ is the dimension of the affine span of the vertices $V$.

Proof Without loss of generality we assume that $\mathbf{p}_{H}$ is a configuration in $\mathbb{R}^{d}=H$, that the cone point $\mathbf{u}$ is in $\mathbb{R}^{d+1}-\mathbb{R}^{d}$, and that the affine span is $\mathbb{R}^{d+1}$. Using the notation of Sect. 4, let $\hat{P}_{H}$ be the augmented configuration matrix for $\mathbf{p}_{H}$, and suppose that $\Omega$ is a stress matrix for a stress for $\mathbf{p}_{H}$. Then $\hat{P}_{H} \Omega=\mathbf{0}$. Transform the configuration for $\mathbf{p}$ in $\mathbb{R}^{d+1}$ so that the cone point $\mathbf{u}$ goes to the point $[1,0, \ldots, 0]$, a point at infinity in homogeneous coordinates (with $(d+2)$ coordinates) as in Sect. 4.

Let $\gamma$ be the 1-by- $|V|$ row vector whose coordinates are the coordinates of the vectors $\mathbf{p}_{i}$ of $\mathbf{p}$ in the extra homogeneous coordinate, and let $O$ be the 1-by- $d$ column vector of zeros. Then, in this homogeneous form, the configuration matrix $Q$ that includes the extra point at infinity is

$$
Q=\left[\begin{array}{cc}
\gamma & 1 \\
\hat{P_{H}} & O
\end{array}\right],
$$

where the last column of $Q$ corresponds to the image of the cone point $\mathbf{u}$. So $Q=$ $A \hat{P}$, and $\hat{P} \tilde{\Omega}=\mathbf{0}$, where $\tilde{\Omega}$ is a stress matrix for the configuration $\mathbf{p}$, and $A$ is a nonsingular $(d+2)$-by- $(d+2)$ matrix.

It is clear from (2) that the $\operatorname{rank}(Q)=\operatorname{rank}\left(\hat{P}_{H}\right)+1$. If $\operatorname{rank}\left(\hat{P}_{H}\right)=d+1$, then the rows of $\hat{P}_{H}$ are a basis for the kernel of $\Omega$, and $\operatorname{rank}(\Omega)=|V|-(d+1)$, the maximum possible. Similarly, if $\operatorname{rank}(Q)=d+2$, then the rows of $Q$ are a basis for the kernel of $\tilde{\Omega}$, and $\operatorname{rank}(\tilde{\Omega})=|V|+1-(d+2)=|V|-(d+1)$, the maximum possible. So if either the rank of $\Omega$ or $\tilde{\Omega}$ is $|V|-(d+1)$, we can choose $\hat{P}_{H}$ or $Q$ to have maximal rank, and both $\Omega$ and $\tilde{\Omega}$ will have rank $|V|-(d+1)$.

This confirms the following result which was conjectured in [4].

Corollary 10 (Global Rigidity Coning) A graph $G$ is generically globally rigid in $\mathbb{R}^{d}$ if and only if the cone graph $G *\{v\}$ is generically globally rigid in $\mathbb{R}^{d+1}$.

Proof Choose a configuration $\mathbf{p} *$ for $G *\{v\}$ that is generic in $\mathbb{R}^{d+1}$ and $G *\{v\}(\mathbf{p} *)$ is infinitesimally rigid, either in $\mathbb{R}^{d+1}$, such that its projection $G\left(\mathbf{p}_{H}\right)$ in $\mathbb{R}^{d}$ is infinitesimally rigid in $\mathbb{R}^{d}$. Theorem 8 implies that they are both infinitesimally rigid. Then apply Theorem 9 and Theorem 5. 


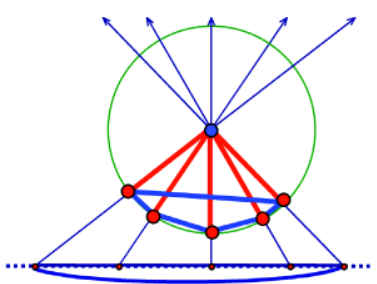

(a)

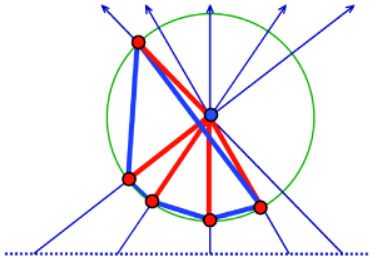

(b)

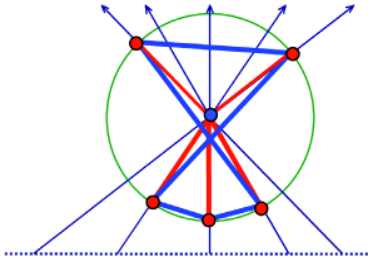

(c)

Fig. 3 Different frameworks on the sphere can share the same lines from the center, the same projection, and have the same global rigidity

\section{Global Rigidity on the Sphere}

For this section, we assume that the cone vertex is the origin $\mathbf{0}$ and that the other points are distinct, with no edge on a line through the origin. There is a connection between the behavior of cone frameworks $G *\{v\}(\mathbf{p} *)$ in $\mathbb{R}^{d+1}$ and the behavior of the associated framework $G\left(\mathbf{p}_{s}\right)$ on the sphere where all vertices of $\mathbf{p}$ have been projected from $\mathbf{0}$ onto the unit sphere $\mathbb{S}^{d}$ centered at $u$. (Geometrically, we extend the line $\mathbf{0} \mathbf{p}_{i}$ and intersect this with the sphere (Fig. 3).)

We already know that this projection from $\mathbb{R}^{d+1}$ to the sphere $\mathbb{S}^{d}$ takes infinitesimally rigid frameworks to infinitesimally rigid frameworks on the sphere $[17,20]$. We now show that this projection to the sphere also takes globally rigid frameworks to globally rigid frameworks.

Of course, such configurations on the sphere are not generic, as frameworks in $\mathbb{R}^{d+1}$, and we will give a geometric argument for the key equivalence. That is, if any cone with the cone vertex at $\mathbf{0}$ is globally rigid, then the cone with all cone lengths of size one is globally rigid (and vice versa) (see below).

Proposition 11 A cone framework with cone vertex at the origin $G *\{v\}(\mathbf{p} *)$ is globally rigid in $\mathbb{R}^{d+1}$ if and only if the spherical framework $G\left(\mathbf{p}_{\mathbb{S}}\right)$ is globally rigid in $\mathbb{S}^{d}$.

Proof We note that any congruence of the $d$-sphere is also a restriction of a unique congruence of $\mathbb{R}^{d+1}$, which fixes the origin (the center of the sphere). Conversely, any congruence of $\mathbb{R}^{d+1}$ which fixes the origin (the center of the sphere) is automatically a congruence of the sphere, when restricted to points on the sphere.

Assume that $G *\{v\}(\mathbf{p} *)$ is not globally rigid, with a second noncongruent realization $G *\{v\}(\mathbf{q} *)$, with the same cone vertex. Then $\mathbf{q}_{s}$ and $\mathbf{p}_{s}$ are also noncongruent, and the radial bars $\mathbf{0} \mathbf{p}_{i}, \mathbf{0} \mathbf{p}_{j}$, and $\mathbf{0} \mathbf{q}_{i}, \mathbf{0} \mathbf{q}_{j}$ have equal lengths. The third sides of the triangle $\mathbf{p}_{i} \mathbf{p}_{j}, \mathbf{q}_{i} \mathbf{q}_{j}$ also have equal length bars $\left(\mathbf{p}_{s}\right)_{i}\left(\mathbf{p}_{s}\right)_{j},\left(\mathbf{q}_{s}\right)_{i}\left(\mathbf{q}_{s}\right)_{j}$. This then generates equal lengths for any bar on the sphere. We conclude that $G\left(\mathbf{p}_{s}\right)$ and $G\left(\mathbf{q}_{s}\right)$ are bar-equivalent and are not congruent. Therefore $G\left(\mathbf{p}_{s}\right)$ is not globally rigid on the sphere.

Conversely, assume that $G\left(\mathbf{p}_{s}\right)$ is not globally rigid on the $d$-sphere, with a noncongruent bar-equivalent $G\left(\mathbf{q}_{s}\right)$. Moving vertices along rays to $G *\{v\}(\mathbf{p} *)$ specifies new distances to the cone point (center of the sphere) that are also bar-equivalent to 
$G *\{v\}(\mathbf{q} *)$. Any congruence of $\mathbf{p} *$ and $\mathbf{q} *$ will give a corresponding congruence of $\mathbf{p}_{s}$ and $\mathbf{q}_{s}$. We conclude that $G *\{v\}(\mathbf{p} *)$ is not globally rigid.

From this geometric result, we have a generic result. We have the technical difficulty that if any two points (or any one point on the unit sphere) of any configuration lie on any sphere through the origin, the configuration is not generic. This is inconvenient. So we say that any configuration in $\mathbb{S}^{d}$ that is the central projection of a generic configuration in $\mathbb{R}^{d+1}$ is quasi-generic in $\mathbb{S}^{d}$.

From Proposition 11, we also note that if a configuration $\mathbf{p}$ is generic in $\mathbb{R}^{d+1}$, then $\mathbf{p}_{s}$ is quasi-generic on the $d$-sphere. The converse is not quite true, but if $\mathbf{p}_{s}$ is quasigeneric on the $d$-sphere, then some of the cones over $\mathbf{p}$ are generic. In particular, without any additional work, we know that global rigidity in $\mathbb{S}^{d}$ is also a quasi-generic property.

Combining this with Corollary 10, we have the desired transfer between Euclidean space and spherical space of the same dimension.

Theorem 12 (Spherical Transfer of Generic Global Rigidity) A graph $G$ is generically globally rigid in $\mathbb{R}^{d}$ if and only if $G$ is quasi-generically globally rigid in the sphere $\mathbb{S}^{d}$.

\section{The Pogorelov Map}

Note that Examples 8.3 and 8.6 show that for nongeneric configurations, a framework may be globally rigid in the plane, but not when lifted up to the sphere, and a framework may be globally rigid on the sphere with the projection not globally rigid in the plane. The generic form of this result is the best possible.

It is interesting to observe that Theorem 12 can be shown by methods of Pogorelov [16], and these methods also show how to do a similar transfer to hyperbolic space. The idea is that there is a function $\tilde{T}: \mathbb{S}_{+}^{|V| d} \times \mathbb{S}_{+}^{|V| d} \rightarrow \mathbb{R}^{|V| d} \times \mathbb{R}^{|V| d}$ that takes a pair of edge-equivalent configurations $\mathbf{p}, \mathbf{q}$ in $\mathbb{S}_{+}^{|V| d}$, the half sphere, to a corresponding pair of edge-equivalent configurations in $\mathbb{R}^{|V| d}$ such that $G(\mathbf{p})$ is equivalent to $G(\mathbf{q})$ if and only if the corresponding image configurations are equivalent in $\mathbb{R}^{|V| d}$. This can be used to show Theorem 12, which we outline here.

Let $\mathbf{n}$ be the vector $\mathbf{n}=(\mathbf{0}, 1)$ in $\mathbb{R}^{d} \times \mathbb{R}^{1}=\mathbb{R}^{d+1}$, the north pole of the sphere $\mathbb{S}^{d}$, and define $\mathbb{S}_{+}^{d}=\left\{\mathbf{v} \in \mathbb{S}^{d} \mid\langle\mathbf{v}, \mathbf{n}\rangle>0\right\}$, where $\langle\cdot, \cdot\rangle$ is the standard inner product in $\mathbb{R}^{d+1}$. Then define $T: \mathbb{S}_{+}^{d} \times \mathbb{S}_{+}^{d} \rightarrow \mathbb{R}^{d}$ by

$$
T\left(\mathbf{p}_{1}, \mathbf{q}_{1}\right)=\left(\mathbf{p}_{1}-\left\langle\mathbf{n}, \mathbf{p}_{1}\right\rangle \mathbf{n}\right) /\left\langle\mathbf{n}, \mathbf{p}_{1}+\mathbf{q}_{1}\right\rangle
$$

for $\left(\mathbf{p}_{1}, \mathbf{q}_{1}\right) \in \mathbb{S}_{+}^{d} \times \mathbb{S}_{+}^{d}$, where $\mathbb{R}^{d}=\mathbb{R}^{d} \times \mathbf{0} \subset \mathbb{R}^{d+1}$. Then the map $\tilde{T}$ is defined by $\tilde{T}(\mathbf{p}, \mathbf{q})=\left(T_{1}(\mathbf{p}, \mathbf{q}), T_{2}(\mathbf{p}, \mathbf{q})\right)=\left(T_{1}(\mathbf{p}, \mathbf{q}), T_{1}(\mathbf{q}, \mathbf{p})\right)$, where

$$
T_{1}(\mathbf{p}, \mathbf{q})=\left(T\left(\mathbf{p}_{1}, \mathbf{q}_{1}\right), \ldots, T\left(\mathbf{p}_{|V|}, \mathbf{q}_{|V|}\right)\right),
$$

and $T$ is defined by (3). For a single pair of vertices $\left(\mathbf{p}_{1}, \mathbf{q}_{1}\right), \tilde{T}$ is a central projection into a $2 d$-dimensional hyperplane orthogonal to $(\mathbf{n}, \mathbf{n})$, followed by projection 
parallel to $(\mathbf{n}, \mathbf{0})$ or $(\mathbf{0}, \mathbf{n})$ into $\mathbb{R}^{d} \times \mathbb{R}^{d}$. It is a straightforward, but tedious, exercise to verify that $\left\|T\left(\mathbf{p}_{i}, \mathbf{q}_{i}\right)-T\left(\mathbf{p}_{j}, \mathbf{q}_{j}\right)\right\|=\left\|T\left(\mathbf{q}_{i}, \mathbf{p}_{i}\right)-T\left(\mathbf{q}_{j}, \mathbf{p}_{j}\right)\right\|$ if and only if $\left\|\mathbf{p}_{i}-\mathbf{p}_{j}\right\|=\left\|\mathbf{q}_{i}-\mathbf{q}_{j}\right\|$, where $\|\cdot\|^{2}=\langle\cdot, \cdot\rangle$. Thus, for any graph $G$ and configurations $\mathbf{p}, \mathbf{q}$ in $\mathbb{S}_{+}^{d}, G(\mathbf{p})$ is edge equivalent to $G(\mathbf{q})$ if and only $G\left(T_{1}(\mathbf{p}, \mathbf{q})\right)$ is edge equivalent to $G\left(T_{2}(\mathbf{p}, \mathbf{q})\right)$. It is also straightforward to verify that $\tilde{T}$ is a homeomorphism. Namely it is one-to-one and onto.

Suppose that $\mathbf{p}$ is a generic configuration in $\mathbb{R}^{d}$ and $G$ is not generically globally rigid in $\mathbb{R}^{d}$. By the results of [6] and [10], for an open neighborhood of $U$ of $\mathbf{p}$ in $\mathbb{R}^{d|V|}$, there is an injection $f: U \rightarrow \mathbb{R}^{d|V|}$ such that $G(\mathbf{p})$ is edge-equivalent but not congruent to $G(f(\mathbf{p}))$ for all $\mathbf{p} \in U$. Then by the remarks above, $\tilde{T}^{-1}(\mathbf{p},(f(\mathbf{p})) \in$ $\mathbb{S}_{+}^{d} \times \mathbb{S}_{+}^{d}$ consists of two $G$ edge-equivalent but not congruent configurations in $\mathbb{S}_{+}^{d}$. It is also easy to verify that projection onto the first coordinate of $\tilde{T}^{-1}(\{\mathbf{p}, f(\mathbf{p})) \mid$ $\mathbf{p} \in U\})$ is open in $\mathbb{S}_{+}^{d}$. This means that quasi-generic configurations in $\mathbb{S}_{+}^{d}$ are not globally rigid for the graph $G$.

Similarly, for any quasi-generic configuration $\mathbf{p}$ in $\mathbb{S}^{d}$, by replacing some vertices by their antipodes, we can assume that the configuration lies in $\mathbb{S}_{+}^{d}$. If it is not globally rigid in $\mathbb{S}_{+}^{d}$, an argument similar to the one in [6] shows that there is a homeomorphism from an open neighborhood $U$ of $\mathbf{p}$ to another open subset of $\mathbb{S}^{d|V|}$ as in the Euclidean case. We can then use the Pogorelov map $\tilde{T}$ in the opposite direction, to show that $G$ is not generically globally rigid in $\mathbb{R}^{d}$, finishing this proof of Theorem 12.

It is also possible to use this idea to prove that a graph $G$ is generically globally rigid in $\mathbb{R}^{d}$ if and only if $G$ is quasi-generically globally rigid in the hyperbolic space $\mathbb{H}^{d}$. Here one uses the same formulas (3) and (4), but the inner product $\langle\cdot, \cdot\rangle$ is given by the indefinite inner product where $\mathbb{H}^{d}=\{\mathbf{v} \mid\langle\mathbf{v}, \mathbf{v}\rangle=-1\}$ and $\|\mathbf{v}-\mathbf{w}\|^{2}=\langle\mathbf{v}-$ $\mathbf{w}, \mathbf{v}-\mathbf{w}\rangle$ defines the distance between $\mathbf{v}$ and $\mathbf{w}$ in $\mathbb{H}^{d}$.

This completes a circle of ideas, concerning cones, projections, and averaging as in Sect. 8, which were used in [17] and [18] to understand Pogorelov's map.

\section{Examples and a Further Criterion}

We present some examples that are illustrative or provide counterexamples to natural conjectures one might be inclined to make. Figure 4 will be used for several examples. Consider the cone over a cycle, as in Fig. 4. Let $\theta_{i}$ for $i=1, \ldots, n$ be the directed internal angle between successive edges adjacent to the cone point, indices modulo $n$. The plane configuration is globally rigid if and only if, for all $\epsilon_{i}= \pm 1, i=1, \ldots, n$, such that $\sum_{i}^{n} \epsilon_{i} \theta_{i}=0(\bmod 2 \pi)$, all the $\epsilon_{i}$ are equal.

Example 8.1 The cone on the rectangle in Fig. 4 is infinitesimally rigid in the plane, and it has a stress matrix with rank $2=5-3$. This is a certificate showing that the underlying graph is generically globally rigid in the plane, but not in that configuration.

Example 8.2 It is easy to see that the cone in Fig. 4 projects to a configuration in the line. The configuration in the line is globally rigid in the line, but again the cone is not globally rigid in the plane. 


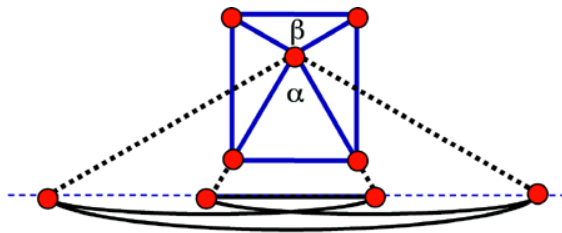

(a)

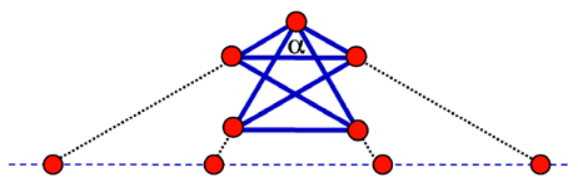

(b)

Fig. 4 (a) This is a cone in the plane that is not globally rigid, although the projection is globally rigid on the line. Here $\alpha=60^{\circ}, \beta=120^{\circ}$, and the other two angles at the cone vertex are $90^{\circ}$. (b) This is a second realization with the same edge lengths

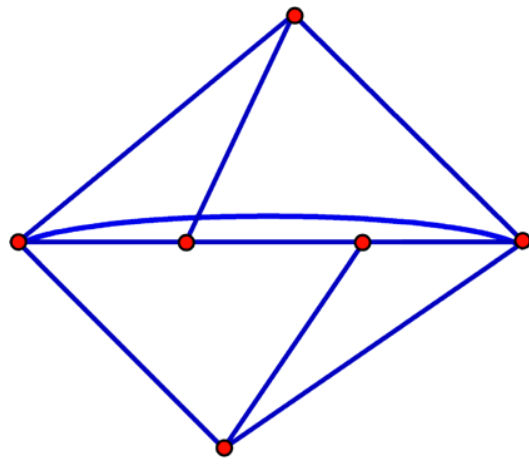

(a)

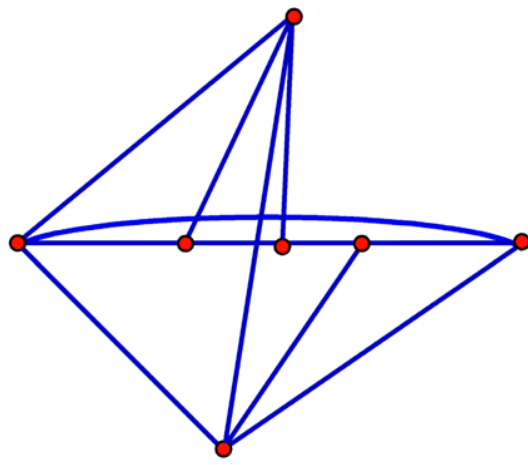

(b)

Fig. 5 (a) The four horizontal vertices form a cycle in a line. The long edge in that cycle is shown as an arc, so that the different bars will be visible. This is not globally rigid, though the graph is generically globally rigid. (b) The five horizontal vertices form a cycle on the line, and its stress matrix has lower rank. The framework is isostatic and globally rigid, and this guarantees that the graph is generically globally rigid

Example 8.3 Suppose that the configuration of the cone in Fig. 4 is perturbed slightly in the plane, say to a generic configuration. That generic configuration will be globally rigid in the plane. However, by applying an affine shear that only stretches or shrinks in the vertical direction it is possible to increase or decrease the angles $\alpha$ and $\beta$ together so that $\alpha+\beta=180^{\circ}$ again, and thus the cone returns to being not globally rigid in the plane in the new, nongeneric configuration.

This shows that global rigidity, even starting at a generic configuration which is not an affine invariant. By way of contrast, at a generic configuration, rigidity (and equivalently infinitesimal rigidity) is an affine invariant, and more strongly, a projective invariant.

Example 8.4 One must be careful about how to apply Theorem 5. Just because a given configuration is infinitesimally rigid and the graph is generically globally rigid, it does not mean that there is necessarily a stress matrix of maximal rank. Nongeneric configurations can sneak in unexpected ways. Figure 5a shows an example of an infinitesimally rigid framework in the plane that is generically globally rigid with a one-dimensional stress space, where the nonzero stress matrices are all of rank 
$2<6-(2+1)=|V|-(d+1)$. This is because the members adjacent to the top and bottom vertices all have 0 stress, and because the middle degree three vertices in the line segment are adjacent to two collinear bars, and so the bar going to the top or bottom vertex must have 0 stress. Since the other bars at the top and bottom vertices are not collinear, their stresses must be 0 as well. So the rank of the stress matrix $\Omega$ must be the same as the rank of the 4-by-4 submatrix corresponding to the four vertices on the horizontal line. That rank is 2 .

Example 8.5 The framework in Fig. 5b also has only the stress on the collinear polygon-which gives a stress matrix of rank $3<7-(2+1)=|V|-(d+1)$. However, the framework is infinitesimally rigid and globally rigid. We will see below that these properties are sufficient to prove that the graph is generically globally rigid.

Example 8.6 One might ask if there is a framework, $G(\mathbf{p})$, where there is a generic configuration with some stress matrix of not maximal rank. Such a simple example is when $G$ is the complete graph $K_{5}$ in the plane. Generically $K_{5}$ has a stress in threespace. A generic configuration in three-space projects to a generic configuration in the plane, and since the affine span of the $K_{5}$ in three-space is three-dimensional, the rank of the associated stress matrix will not be maximal for this stress. Of course, by Theorem 3, there is another stress which has a stress matrix of maximal rank.

Example 8.7 It is possible to provide an example of a cone that is globally rigid in the plane that projects to a cycle of four points in the line, which are not globally rigid in the line. For example, permute the cyclic graph whose configuration is $P=$ $[0,2,3,1]$ to $[1,3,2,0]$ on the line. If the cone point is chosen generically, the cone will not be globally rigid in the plane. Figure 6 shows this.

Example 8.8 The framework on $K_{3,3}$ in Fig. $6 \mathrm{~b}$ is globally rigid in this special configuration, but it is not infinitesimally rigid when it has a stress. The graph is not

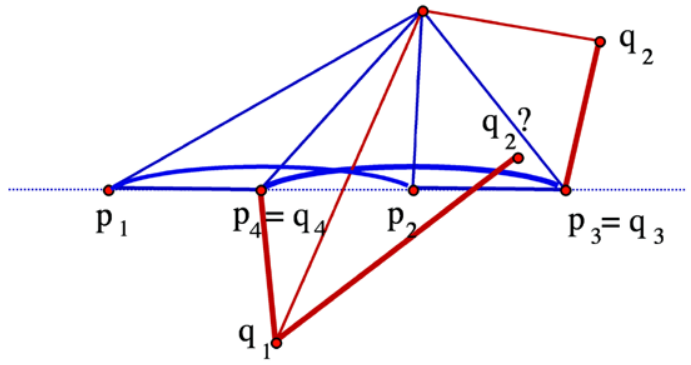

(a)

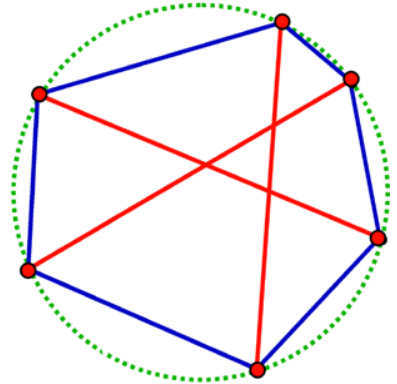

(b)

Fig. 6 (a) A cycle in the line that is not globally rigid in the line. But the cone over this graph is globally rigid in the plane. The two thick edges are the same length and must connect $\mathbf{q}_{1}$ with $\mathbf{q}_{2}$ to complete the alternate realization, and they do not meet. (b) $K_{3,3}$ on an ellipse has one stress with tension on boundary hexagon and compression on the three interior bars. In this configuration, the framework is globally rigid in all dimensions 
generically globally rigid because it is generically isostatic and thus not redundantly rigid.

As suggested by Example 8.5, there is one further connection to explore. Given a framework $G(\mathbf{p})$ which is infinitesimally rigid and globally rigid in $\mathbb{R}^{d}$ but does not have a stress of rank $|V|-(d+1)$ (Example 8.8), do we know that the graph is generically globally rigid in $\mathbb{R}^{d}$ ? The following result answers this in a strong way.

Theorem 13 Given a framework $G(\mathbf{p})$ which is globally rigid and infinitesimally rigid in $\mathbb{R}^{d}$, there is an open neighborhood $N_{\mathbf{p}}$ such that for $\mathbf{q} \in N_{\mathbf{p}} . G(\mathbf{q})$ are all globally rigid and infinitesimally rigid.

Proof If there are fewer than $d$ vertices, the assumption of infinitesimal rigid guarantees that this is a complete graph, with joints affinely independent. The theorem is clearly true for such complete graphs.

Assume that $G(\mathbf{p})$ does not have an open neighborhood $N_{\mathbf{p}}$ in which all frameworks are globally rigid, $|V| \geq n$. Then there is a convergent sequence $G\left(\mathbf{p}^{k}\right)$ of nonglobally rigid frameworks, converging to $G(\mathbf{p})$.

For each of the frameworks in this sequence, take one of the other bar-equivalent realizations: $G\left(\mathbf{q}^{k}\right)$. We can select one vertex $v_{0}$, and by translation, bring each $G\left(\mathbf{q}^{k}\right)$ to have $\mathbf{q}_{0}^{k}=\mathbf{p}_{0}$. Then, by compactness, there is a convergent subsequence $G\left(\mathbf{q}^{m}\right)$ converging to $G(\mathbf{q})$. $G(\mathbf{q})$ must be bar-equivalent to the limit of the corresponding $G\left(\mathbf{p}^{m}\right)$, which means bar-equivalent to $G(\mathbf{p})$.

If $G(\mathbf{q})$ is not congruent to $G(\mathbf{p})$, it contradicts the assumption that $G(\mathbf{p})$ was globally rigid. Therefore $G(\mathbf{q})$ is congruent to $G(\mathbf{p})$. If we apply this same congruence to all the $G\left(\mathbf{q}^{m}\right)$, we have two sequences $G\left(\mathbf{r}^{m}\right)$ and $G\left(\mathbf{p}^{m}\right)$, both converging to $G(\mathbf{p})$. Moreover, for each pair, $G\left(\mathbf{r}^{m}\right)$ and $G\left(\mathbf{p}^{m}\right)$ are bar-equivalent.

The general averaging technique shows that $\mathbf{p}^{m}-\mathbf{r}^{m}$ is an infinitesimal motion of the average framework: $G\left(\frac{\mathbf{p}^{m}+\mathbf{r}^{m}}{2}\right)$. Specifically, for each edge $\{i, j\}$ :

$$
\begin{aligned}
& \left(\frac{\mathbf{p}_{i}^{m}+\mathbf{r}_{i}^{m}}{2}-\frac{\mathbf{p}_{j}^{m}+\mathbf{r}_{j}^{m}}{2}\right) \cdot\left[\left(\mathbf{p}_{i}^{m}-\mathbf{r}_{i}^{m}\right)-\left(\mathbf{p}_{j}^{m}-\mathbf{r}_{j}^{m}\right)\right] \\
& \quad=\frac{1}{2}\left[\left(\mathbf{p}_{i}^{m}-\mathbf{p}_{j}^{m}\right)+\left(\mathbf{r}_{i}^{m}-\mathbf{r}_{j}^{m}\right)\right] \cdot\left[\left(\mathbf{p}_{i}^{m}-\mathbf{p}_{j}^{m}\right)-\left(\mathbf{r}_{i}^{m}-\mathbf{r}_{j}^{m}\right)\right] \\
& \quad=\frac{1}{2}\left[\left(\mathbf{p}_{i}^{m}-\mathbf{p}_{j}^{m}\right)^{2}-\left(\mathbf{r}_{i}^{m}-\mathbf{r}_{j}^{m}\right)^{2}\right]=0 .
\end{aligned}
$$

The conclusion that this is 0 follows because of the bar-equivalence of the two frameworks.

Now, since $G(\mathbf{p})$ is infinitesimally rigid, $\mathbf{p}$ affinely spans the space, as do the $\mathbf{p}^{m}$, $\mathbf{r}^{m}$, and $\frac{\mathbf{p}^{m}+\mathbf{r}^{m}}{2}$ for sufficiently large $m$. If we repeat this calculation for pairs that are not edges, then the fact that $\mathbf{p}^{m}$ and $\mathbf{r}^{m}$ are not congruent means that some distance is being changed and that some pair has a nonzero strain (dot product) for this motion. When $\mathbf{p}^{m}$ and $\mathbf{r}^{m}$ are not congruent, then $\mathbf{p}^{m}-\mathbf{r}^{m}$ is a nontrivial infinitesimal motion.

This means that the rank of the rigidity matrix for each of the frameworks $G\left(\frac{\mathbf{p}^{m}+\mathbf{r}^{m}}{2}\right)$ is lower than required for infinitesimal rigidity. Since both $\mathbf{p}^{m}$ and $\mathbf{r}^{m}$ 
converge to $\mathbf{p}$, so does $\frac{\mathbf{p}^{m}+\mathbf{r}^{m}}{2}$. We conclude that $G(\mathbf{p})$ is the limit of frameworks which are not infinitesimally rigid, and therefore is not infinitesimally rigid. This contradiction completes the proof.

Since every open neighborhood contains generic configurations, we have the following corollary, which gives an additional certificate which guarantees generic global rigidity.

Corollary 14 If $G(\mathbf{p})$ is globally rigid and infinitesimally rigid in $\mathbb{R}^{d}$, then $G$ is generically globally rigid in $\mathbb{R}^{d}$.

We call any framework $G(\mathbf{p})$ which provides this certificate (is infinitesimally rigid and globally rigid) strongly rigid.

Corollary 15 If there is one strongly rigid framework $G(\mathbf{p})$ in $\mathbb{R}^{d}$, then the set of all configurations $\mathbf{q}$ making $G(\mathbf{q})$ strongly rigid is open and dense in $\mathbb{R}^{d}$, and includes all generic configurations.

Suppose that a graph $G$ has no configuration $\mathbf{p}$ where $G(\mathbf{p})$ is strongly rigid. In this case, the set of globally rigid frameworks is thin-of co-dimension at least 1 . Provided that the graph is at least 2-connected, there will be some globally rigid frameworks, for example, putting all the vertices in a line. However, none of these globally rigid frameworks can be infinitesimally rigid. They must also have a stress, by the original results of Hendrickson.

The concept of strongly rigid frameworks applies to spherical frameworks, as a guarantee of an open dense subset, and of generic global rigidity, since it applies to the cones to the origin.

The proof of Theorem 13 applies in all projective metrics, such as hyperbolic geometry, since the averaging process works in all these metrics [18]. So the concept of strong rigidity extends to these other metrics. We also know that infinitesimal rigidity transfers for projectively equivalent configurations, and therefore generically, to these other metrics [17].

From these results, examples, and observations for cones and spherical frameworks, we have the following:

1. By Theorems 8 and 9 , a cone framework $G *\{v\}(\mathbf{p} *)$ is a certificate for generic global rigidity of $G *\{v\}$ in $\mathbb{R}^{d+1}$ if and only if $G\left(\mathbf{p}_{H}\right)$ is a certificate for generic global rigidity of $G$ in $\mathbb{R}^{d}$ if and only if $G\left(\mathbf{p}_{\mathbb{S}}\right)$ is a certificate for generic global rigidity of $G$ in $\mathbb{S}^{d}$. The concept and properties of a certificate for generic global rigidity in $\mathbb{S}^{d}$ are well defined.

2. By Theorems 8 and $11, G *\{v\}(\mathbf{p} *)$ is strongly rigid in $\mathbb{R}^{d+1}$ if and only if $G\left(\mathbf{p}_{\mathbb{S}}\right)$ is strongly rigid in $\mathbb{S}^{d}$.

3. $G *\{v\}(\mathbf{p} *)$ may be globally rigid in $\mathbb{R}^{d+1}$ (equivalently $G\left(\mathbf{p}_{\mathbb{S}}\right)$ is globally rigid $\mathbb{S}^{d}$ ) even if $G\left(\mathbf{p}_{H}\right)$ is not globally rigid $\mathbb{R}^{d}$ (Example 8.2).

4. $G\left(\mathbf{p}_{H}\right)$ may be globally rigid $\mathbb{R}^{d}$ even if $G *\{v\}(\mathbf{p} *)$ is not globally rigid in $\mathbb{R}^{d+1}$ (equivalently $G\left(\mathbf{p}_{\mathbb{S}}\right)$ is not globally rigid $\mathbb{S}^{d}$ ) (Example 8.7). 
For a general graph $G$, we have the following breakdown of possibilities at particular frameworks.

Case 8.1 For generic configurations $\mathbf{p}$, there are three possibilities:

(a) $G(\mathbf{p})$ is not infinitesimally rigid and thus not rigid and not globally rigid.

(b) The framework $G(\mathbf{p})$ has a stress with rank of the stress matrix equal to $|V|-(d+1)$. The framework $G(\mathbf{p})$ is globally rigid in $\mathbb{R}^{d}$, and all generic configurations are globally rigid in $\mathbb{R}^{d}$.

(c) All stress matrices have a rank $|V|-(d+2)$ or less. This framework is not globally rigid in $\mathbb{R}^{d}$, and no generic configuration makes $G$ globally rigid in $\mathbb{R}^{d}$.

Case 8.2 For nongeneric realizations $\mathbf{p}$ in $\mathbb{R}^{d}$, there are many possibilities:

(a) The framework $G(\mathbf{p})$ is a certificate for generic global rigidity (has a stress matrix with rank $|V|-(d+1)$ and is infinitesimally rigid). In this case, the graph $G$ is generically globally rigid in $\mathbb{R}^{d}$ (Theorem 5).

(b) The framework $G(\mathbf{p})$ is strongly rigid (is infinitesimally rigid and globally rigid). The configuration has an open neighborhood of globally rigid frameworks, and the graph $G$ is generically globally rigid in $\mathbb{R}^{d}$ (Theorem 13 and Corollary 14).

(c) The framework $G(\mathbf{p})$ is not infinitesimally rigid. We learn nothing about the generic global rigidity of $\mathrm{G}$, regardless of whether $G(\mathbf{p})$ is globally rigid or not, and regardless of whether $G(\mathbf{p})$ has a stress with a stress matrix of rank $|V|-(d+1)$ or not (Examples 8.1-8.8).

\section{Other Conjectures}

This analysis brings up a further question: Is there an easily calculated quantity that will determine global rigidity of $G(\mathbf{p})$ at the given configuration with probability 1 ? By way of contrast, for rigidity, such a quantity is infinitesimal rigidity, and the calculation of the rank of the rigidity matrix for the given configuration. For global rigidity, the examples show that even having infinitesimal rigidity and a stress with stress matrix rank $|V|-(d+1)$ is not sufficient for that configuration, though it is a certificate for generic global rigidity of the graph, as discussed in Sect. 4. More generally, the Tarski-Seidenberg elimination theory shows that there must exist a finite list of polynomial equations [7] — but this does not provide any constructive process for identifying them.

Vertex-splitting is another general technique for extending generically rigid frameworks to larger generically rigid frameworks, in each dimension [21, 22]. Recently, Jordán and Szabadka [15] have proven that vertex splitting with both vertices of degree $\geq 3$ preserves global rigidity for all graphs in the plane. The following was conjectured in [4].

Conjecture 16 (Global Rigidity and Vertex Splitting) If $G$ is a generically globally rigid graph in $\mathbb{R}^{d}, n \geq 3$, and $G^{\prime}$ is generated from $G$ by vertex-splitting for $d$-space, so that each of the split vertices has valence at least $d+1$, then $G^{\prime}$ is generically globally rigid in $\mathbb{R}^{d}$. 
One difficulty with replicating the edge-splitting proof in [7] is to show that vertexsplitting on a redundantly rigid graph generates a redundantly rigid graph. In particular, what is missing is that the edge joining the two split copies of the vertex is redundant!

In the following, we go out on a limb.

Conjecture 17 In dimension $3, K_{5,5}$ is the only vertex 4-connected generically redundantly rigid graph that is not generically globally rigid.

More generally, we conjecture that, for any dimension $n \geq 3$, the set of redundantly rigid, $(d+1)$-connected graphs which are not generically globally rigid is finite. Related to this is whether there is such a generically redundantly rigid, vertex $(d+1)$-connected, but not generically globally rigid graph, that has a complete graph $K_{d+1}$ as a subgraph. If such a graph exists, then there might be a way of building several such examples.

\section{References}

1. Anderson, B.D.O., Belhumeur, P., Eren, T., Goldenberg, D., Morse, A.S., Whiteley, W., Yang, Y.: Graphical properties of easily localizable sensor networks. Wirel. Netw. 15(2), 177-191 (2009)

2. Bolker, E.D., Roth, B.: When is a bipartite graph a rigid framework? Pac. J. Math. 90(1), 27-44 (1980)

3. Berg, A., Jordán, T.: A proof of Connelly's conjecture on 3-connected circuits of the rigidity matroid. J. Comb. Theory, Ser. B 88, 77-97 (2003)

4. Cheung, M., Whiteley, W.: Transfer of global rigidity results among dimensions: graph powers and coning. Preprint, York University, Revised 2008

5. Connelly, R.: Rigidity and energy. Invent. Math. 66(1), 11-33 (1982)

6. Connelly, R.: On generic global rigidity. In: Applied Geometry and Discrete Mathematics. DIMACS Ser. Discrete Math, Theoret. Comput. Sci., vol. 4, pp. 147-155. AMS, Providence (1991)

7. Connelly, R.: Generic global rigidity. Discrete Comput. Geometry 33, 549-563 (2005)

8. Connelly, R., Jordán, T., Whiteley, W.: Generic global rigidity of body bar frameworks. Preprint (2009)

9. Eren, T., Goldenberg, D., Whiteley, W., Morse, A., Anderson, B.D.O., Belhumeur, P.: Rigidity, computation, and randomization in network localization. In: Proceedings of the International Annual Joint Conference of the IEEE Computer and Communications Societies (INFOCOM), Hong Kong, March 2004, pp. 2673-2684

10. Gortler, S., Healy, A., Thurston, D.: Characterizing generic global rigidity. arXiv:0710.0907v1 (2007)

11. Graver, J.: Counting on Frameworks: Mathematics to Aid the Design of Rigid Structures. Dolciani Mathematical Expositions, vol. 25. The Mathematical Association of America, Washington (2001)

12. Hendrickson, B.: Conditions for unique graph realizations. SIAM J. Comput. 21, 65-84 (1992)

13. Jackson, B., Jordán, T.: Connected rigidity matroids and unique realization graphs. J. Comb. Theory B 94, 1-29 (2005)

14. Jackson, B., Jordán, T., Szabadka, Z.: Globally linked pairs of vertices in equivalent realizations of graphs. Discrete Comput. Geom. 35(3), 493-512 (2006)

15. Jordán, T., Szabadka, Z.: Operations preserving the global rigidity of graphs and frameworks in the plane. Comput. Geom. 42(6-7), 511-521 (2009)

16. Pogorelov, A.V.: A Study of Surfaces in an Elliptic Space, (English Translation of the Russian Title "Nekotorye Voprosy Teorii Poverkhnostei") Hindustan Publishing Corporation (India) 6-U.B., Jawahar Nagar, DELHI-6 (1964)

17. Saliola, F., Whiteley, W.: Some notes on the equivalence of first-order rigidity in various geometries. Preprint Department of Mathematics, York University (2002)

18. Saliola, F., Whiteley, W.: Averaging and equivalent frameworks: transfer among various geometries. Draft, Department of Mathematics, York University (2005) 
19. Tay, T.-S., Whiteley, W.: Generating isostatic frameworks. Structural Topology 11, $20-69$ (1985)

20. Whiteley, W.: Cones, infinity and one-story buildings. Struct. Topol. 8, 53-70 (1983)

21. Whiteley, W.: Vertex splitting in isostatic frameworks. Struct. Topol. 16, 23-30 (1991)

22. Whiteley, W.: Matroids from discrete geometry, In: Bonin, J.E., Oxley, J.G., Servatius, B. (eds.) Matroid Theory. American Mathematical Society, Contemporary Mathematics, vol. 197, pp. 171-313 (1996)

23. Whiteley, W.: Rigidity and Scene Analysis, In: Goodman, J., O'Rourke, J. (eds.) Handbook of Discrete and Computational Geometry, 2nd edn., pp. 1327-1354 (2004) 